\section{Response to Letter To THE Editor}

\section{RE: Intracranial Pressure Monitors in Traumatic Brain Injury: A Systematic Review. Can J Neurol Sci. 2013;40: 433-434.}

We thank Dr. Figaji for his thoughtful commentary ${ }^{1}$ on our recent systematic review addressing intracranial pressure monitors in traumatic brain injury (TBI $)^{2}$. We completely concur with his eloquent summary of the limitations of the literature. Although Dr. Figaji takes slight issue with our contention that in order to improve outcome, intracranial pressure (ICP) monitoring "... must be acted upon in a standardized and reproducible manner", we feel our statement is in fact congruous with his central thesis. This is illustrated nicely in his discussion: elevated ICP is simply a number which may represent various pathophysiologic mechanisms which themselves may have very different treatment approaches.

Intracranial pressure monitoring may be considered analogous to the pulmonary artery catheter (PAC) which generates objective physiologic information which the clinician must then interpret to both diagnose the problem and prescribe a remedy. However, the "right" response is predicated on correct interpretation by clinicians, who perform poorly in this regard ${ }^{3}$. Previous trials on PAC use have been criticized for both lack of training of study personnel on variable interpretation and lack of treatment algorithms in response to PAC data ${ }^{4}$. Because interventions (in this case ICP monitors) generally have small treatment effects, standardization of clinical decisions is requisite for rigorous scientific evaluation ${ }^{5}$. Dr. Figaji illustrates the challenges with developing a standardized algorithm to approach ICP monitoring. However, standardization of care has resulted in improved outcomes in other complex systems, including patients with $\mathrm{TBI}^{6}$. Hopefully neuromonitoring will lead to an improved understanding of the pathophysiologic mechanisms underlying our this complex disease process. As always, the goal is to improve care for our patients, something we can all agree on.

Donald E. G. Griesdale ${ }^{1,3-5}$, Asher A. Mendelson ${ }^{1}$, Chris Gillis ${ }^{2}$, William R. Henderson ${ }^{1,3}$, Juan J. Ronco ${ }^{1,3}$, Vinay Dhingra ${ }^{1,3}$

University of British Columbia ${ }^{1,2}$; Vancouver General Hospital ${ }^{3,4}$; Vancouver Coastal Health Research Institute ${ }^{5}$ Vancouver, British Columbia, Canada Email:donald.griesdale@vch.ca

\section{REFERENCES}

1. Figaji A. ICP monitoring - interpreting the literature and evaluating practice. Can J Neurol Sci. 2013;40(3):433-4.

2. Mendelson A, Gillis C, Henderson WR, Ronco JJ, Dhingra V, Griesdale DEG. Intracranial pressure monitors in traumatic brain injury: a systematic review. Can J Neurol Sci. 2012 Sep;39(5): 571-6.

3. Squara P, Bennett D, Perret C. Pulmonary artery catheter: does the problem lie in the users? Chest. 2002 Jun;121(6):2009-15.

4. Shure D. Pulmonary-artery catheters-peace at last? NEJM. 2006; 354(21):2273-4

5. Morris AH. Developing and implementing computerized protocols for standardization of clinical decisions. Ann Intern Med. 2000 Mar 7;132(5):373-83

6. Patel HC, Menon DK, Tebbs S, Hawker R, Hutchinson PJ, Kirkpatrick PJ. Specialist neurocritical care and outcome from head injury. Intensive Care Med. 2002 May;28(5):547-53.

\section{To THE EDITOR}

\section{Bacillus Thuringiensis Poisoning Related Acute Transverse Myelitis}

A 25-year-old Asian woman with acute spinal shock was referred to our institution for therapy. She attempted suicide by drinking $250 \mathrm{mg}$ of insecticide containing Bacillus thuringiensis (8000IU/mg) seven days prior to admission. She felt nausea soon after drinking the insecticide, but did not vomit nor did she go to see the doctor until five days later when she felt headache, abdominal pain, sudden weakness on her both legs, and was unable to urinate.

On clinical examination, we found the patient to have normal temperature, pulse, blood pressure and respiration, without mental disorders. The patient experienced a stiff neck. Neurologic evaluation revealed paresis of both lower limbs of grade $0 / 5$ and hypotonia, without Babinskii's sign, knee and ankle deep tendon reflexes were elicited, T10-level sensory deficit, impaired joint position sensation of the lower limbs, and absence of anal sphincter tone. Her cranial nerve functions were normal.
Cerebrospinal fluid (CSF) analysis showed translucent fluid with an opening pressure of $300 \mathrm{cmH}_{2} \mathrm{O}$. The CSF glucose, chloride and adenylate deaminase contents were normal and CSF protein was as high at $98 \mathrm{mg} / \mathrm{dL}$. The CSF leukocyte count was high $\left(220 \times 10^{6} / \mathrm{L}\right.$, of which 97 percent was neutrophils and 3 percent was lymphocytes). The CSF erythrocyte count was also high $\left(440 \times 10^{6} / \mathrm{L}\right)$, however, traumatic puncture was denied and CSF culture for bacteria and fungus showed negative results.

Her serum immunological examination and peripheral blood tests were normal. Tests for antibodies of Coxackie virus, CMV, EBV, HBV, HCV, HIV, HSV1, HSV2, VZV, bacillus tuberculosis, syphilis in serum and CSF, as well as tumor markers in serum revealed no etiological values. Cultures of her urine, stool, and blood were all negative.

Her first spinal magnetic resonance imaging (MRI), taken on the onset day of paralysis, which was five days after her suicide attempt, was normal (Figure 1). Her second spinal MRI, which was taken eight days after her suicide attempt, indicated diffused abnormities in whole spinal cord, along with severe swelling of spinal cord (Figure 2). Cerebral MRI was normal.

Electroencephalogram and visual evoked potentials were normal. Electromyography and peripheral nerve conduction velocity both indicated upper-neuron damage of all four limbs. 